\title{
Visualization of mold filling stages in thermal nanoimprint by using pressure gradients
}

\author{
Helmut Schift ${ }^{\text {a) }}$ \\ Laboratory for Micro- and Nanotechnology, Paul Scherrer Institut, 5232 Villigen PSI, Switzerland \\ Sandro Bellini ${ }^{\text {b) }}$ \\ FIRST Center for Micro- and Nanoscience, ETH Zürich, 8093 Zürich, Switzerland \\ Morten Bo Mikkelsen ${ }^{\text {) }}$ \\ MIC-Department of Micro and Nanotechnology, Technical University of Denmark, 2800 Kongens Lyngby, \\ Denmark \\ Jens Gobrecht ${ }^{\text {d) }}$ \\ Laboratory for Micro- and Nanotechnology, Paul Scherrer Institut, 5232 Villigen PSI, Switzerland
}

(Received 8 June 2007; accepted 17 October 2007; published 10 December 2007)

\begin{abstract}
A method for the visualization of mold filling during a thermoplastic imprint at a microscopic level was developed, which is based on superposition of images of a series of different states of imprint. The animated movie sequence gives an insight into the complex flow of polymer and shows how voids are forming and vanishing during the imprint process. It can therefore be used as a tool for optimization of processes and improvement of stamp design. () 2007 American Vacuum Society. [DOI: 10.1116/1.2806972]
\end{abstract}

\section{INTRODUCTION}

Air bubbles and arrays of voids and mounds with different forms and depths give evidence of the range of dynamic physical effects during the imprint of micropatterns in thin polymer films, ranging from capillary effects, dewetting, trapping of air, and viscous fingering. ${ }^{1}$ Micrographs of partially molded resist often only give a rough indication of what happened during the nanoimprint process (NIL). They are screenshots of the final state in NIL when the polymer reaches its solid state after cooling. ${ }^{2}$ If the imprint process can be stopped at different times, then these screenshots can be combined to a continuous sequence of different states of an imprint, similar to an animated motion picture. This evolution can be observed in a single imprint, when a thickness variation of the stamp and substrate or a pressure gradient results in different states of imprint at different locations. This is because according to Stefan's law [see Eq. (1) for linear protrusions $],{ }^{3}$ for simple setups, pressure $p$ and time $t$ can be interchanged in the formula for the time dependent resist thickness $h(t)$ below a protrusion $\left(h_{0}\right.$ initial polymer height, $\eta_{0}$ the zero shear viscosity, $s$ protrusion width)

$$
\frac{1}{h^{2}(t)}=\frac{1}{h_{0}^{2}}+\frac{2 p}{\eta_{0} s^{2}} t
$$

If a regular structure, e.g., a pillar array with a few microns resolution is imprinted, then micrographs of adjacent, but identical structures with constant increment, can be crossfaded and combined into a movie. In this article we will show single screenshots from such a movie. This movie goes

\footnotetext{
${ }^{a}$ Electronic mail: helmut.schift@psi.ch

${ }^{b)}$ Electronic mail: bellini@ first.ethz.ch

${ }^{c}$ Electronic mail: morten.mikkelsen@mic.dtu.dk

${ }^{d)}$ Electronic mail: jens.gobrecht@psi.ch
}

further than the imprint sequences presented until now because it shows a seamless evolution of a pattern in a defined way. ${ }^{2}$ We will show how this simple method can be applied for the investigation of mold filling in the typical range of optical microscopy and present reasons why-despite all nonlinearity involved-we think that this illustrates important aspects of the actual pattern evolution for thermoplastic NIL. Its main prerequisite is an imprint setup which allows the stopping of the imprint by rapid release of pressure and fast cooling. ${ }^{4}$

\section{EXPERIMENT}

Most of the experimental details, i.e., the setup and the polymers used, are described in more detail in Ref. 4. The resists were structured in an isothermal process, i.e., in a preheated press, at a temperature well above the $T_{g}$ of the polymer. In Ref. 4, already an imprint time of $t_{\text {imprint }}=15 \mathrm{~s}$ was found to be sufficient for complete imprint of regular pillar-like structures with micrometer dimensions, which means that this time is larger than the mold filling time $t_{f}$. For the fast heating and cooling we have used a springloaded adapter for the Süss bond alignment fixture in our Jenoptik HEX03 machine (see Fig. 1). It allows for inserting the stamp/substrate stack without touching the surface of the heated press plates, and by closing the press, the upper plate pushes the fixed stack onto the lower plate, thus causing a fast heating up to the necessary imprint temperature. Upon contact, the full force is applied and imprint begins. At the end of the imprint process, the force is released, the upper plate moves away, and the alignment fixture is pushed upwards by the springs, causing the stack to be separated from the bottom plate by $1 \mathrm{~mm}$, which results in an immediate and fast cooling. 


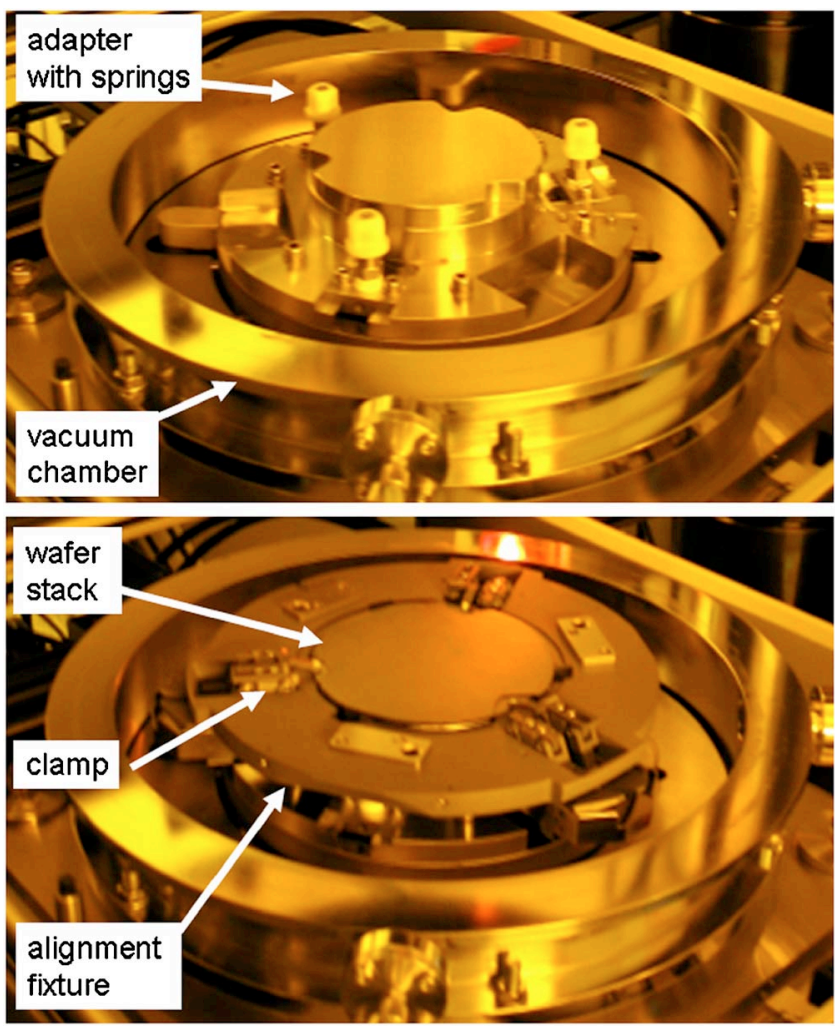

FIG. 1. Adapter at the Jenoptik HEX03 without (top) and with (bottom) Süss alignment fixture, placed in a vacuum chamber (open). During the closing of the press, the stack is pushed down and heats up upon contact. In a similar way the stack is lifted up after imprint with a spring mechanism.

The free standing stack of two $100 \mathrm{~mm}$ wafers is placed onto pedestals extending from the side of the ring-shaped fixture, while the borders are clamped with retractable clamps (see Fig. 1). On top of the stack a $1.5 \mathrm{~mm}$ thick layer of flexible graphite foil is placed as a compliance layer for the equilibration of pressure inhomogeneities.

Using this setup, the thermal mass of the imprint stack is almost restricted to the mass of the stamp and substrate and only small areas of the borders are lost due to the space needed for the clamps of the fixture (around $20 \mathrm{~mm}^{2}$ ). These areas are of particular importance for the current experiments. This is due to the fact that the pressure in these areas is extremely low and only caused by the clamping force. However, the pressure drop from the central plate to these omitted areas is not abrupt, since the limited ability of the substrate and stamp to bend leads to a pressure gradient near the border of the adapter plate. This is because the sinking of the stamp into the polymer in the areas of high pressure causes a strain of the adjacent stamp areas, resulting in bending. A similar effect can be found in areas, where a thickness inhomogeneity, e.g., a dust particle, causes a halo around the defect where the imprint is incomplete. The reason for the incomplete molding in these areas, however, is different from that in the areas with reduced pressure because the stamp is inhibited to sink not by a reduced pressure but by a noncompressible spacer. The distance of the pressure drop from full pressure to nearly zero is anticipated to be in the range of a few millimeters, which corresponds with the observation of typical transition distances. ${ }^{5}$ Heat conduction through the silicon stamp and substrate ensures that the clamped areas are in thermal equilibrium with the areas of high pressure.

For the stamp and substrate $465 \mu \mathrm{m}$ thick silicon wafers were chosen and the stamp structure was a regular array with $4 \mu \mathrm{m}$ sized $(s), 250 \mathrm{~nm}$ deep $\left(h_{r}\right)$ square pillars with a $10 \mu \mathrm{m}$ pitch. Several polymers were tested with the setup. ${ }^{4}$ The sequence we were interested in was obtained in a 300 $\mathrm{nm}$ thick mr-I 8030E from micro resist technology $\mathrm{GmbH}$ with a $T_{g}$ of $115^{\circ} \mathrm{C}$, at $t_{\text {imprint }}=60 \mathrm{~s}$, using a temperature $T_{\text {imprint }}=190^{\circ} \mathrm{C}$, and a maximum force of $F=50 \mathrm{kN}$ (equivalent to $p=64 \mathrm{bar}$ ). According to the process described in Ref. 4, the total imprint time adds up to $135 \mathrm{~s}$, if the pressure buildup and release is taken into account, and the total time of contact with at least one of the heated plates is $195 \mathrm{~s}$. After opening of the press, the $80{ }^{\circ} \mathrm{C}$ hot stack was taken out of the press and further cooled with nitrogen gas, before demolding manually.

The movie is based on 49 adjacent optical micrographs in horizontal direction at one of the areas with reduced pressure, taken with a Nikon Coolpix 990 digital camera on a Leica microscope, at $100 \times$ magnification (see Fig. 2). Each micrograph contains 12 columns of indentations resulting from the imprint of square pillars. From each of these micrographs eight square samples containing $3 \times 3$ indentations are cut out with an increment of one period in horizontal direction. The extraction is done automatically with ImageJ, a free software tool for image processing, which converts the frames into a stack of images. ${ }^{6}$ However, due to the need to align the adjacent micrographs manually, this process is time consuming. The movie generated this way shows the evolution of mold filling at a total length of around $4.9 \mathrm{~mm}$, with a total of 389 frames.

\section{RESULTS}

Measurements of the residual layer thickness $\left(h_{f}\right.$ $=75 \mathrm{~nm})$ and added height of the molded patterns $\left(h_{r}+h_{f}\right.$ $=325 \mathrm{~nm}$ ) indicate that the imprint is complete in all areas of high pressure. However, due to the pressure gradient at the border of the wafer, within a few millimeters distance, different filling states of the clearance between the square pillars can be observed. Figure 2 shows a sequence of micrographs cut out from the movie sequence. It shows that during the sinking of the pillars into the film, the polymer is squeezed out from below the pillars, rises to the top of the cavity, and displaces the voids while flowing around the pillars. It is nonsymmetric which may be attributed to the direction of pressure inhomogeneity. Because of the extreme movement around the pillars it is anticipated that the polymer flow is dominated by capillary forces, which also draw away polymer from nonfilled areas, leading to dewetting type behavior. In contrast to the sequence shown in Ref. 7, where $20 \mu \mathrm{m}$ square cavities were filled, round voids only form at the end of the imprint between the pillars, i.e., when the flowing polymer forces them to minimize surfaces. If fully molded before release, these bubbles vanish, and it is 


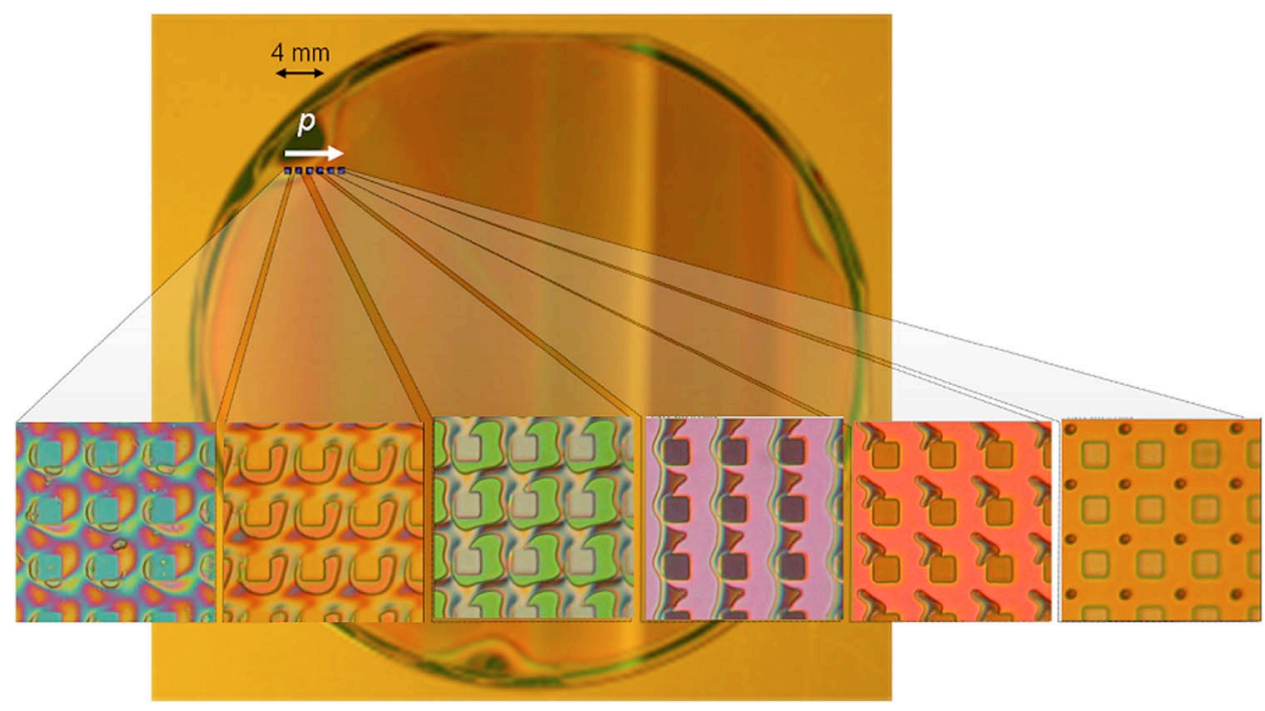

FIG. 2. Photography of a $100 \mathrm{~mm}$ wafer with an imprinted thermoplastic resist. The sequence of six micrographs shows the imprint of regular $4 \mu \mathrm{m}$ pillar stamp structures at different locations. Due to the pressure gradient at the border of the wafer, within a few millimeters different filling states can be observed. Molded structure: hole array of $10 \mu \mathrm{m}$ pitch, $250 \mathrm{~nm}$ deep, resist $300 \mathrm{~nm}$, and imprint time $60 \mathrm{~s}$.

anticipated that the air is entirely dissolved in the polymer. The main finding is that the polymer can flow faster and much more freely than anticipated until now and in a complex way which is far from being random. It varies to some extent for different imprints depending on material, viscosity, and location, but always shows the characteristic capillary bridges, i.e., polymer mounds reaching the top of the cavity, and void forming by surface minimization; e.g. in Fig. 3 a continuous evolution of imprint was observed in a mr-I 9030 $\mathrm{E}$ resist, for which the molding was completed before the curing became significant. ${ }^{4}$ In the black and white representation of the movie sequence areas can be distinguished which represent mounds (high $h$ ), capillary bridges $[h(t)$ $\left.+h_{r}\right]$, nonmolded areas (intermediate $h$ ), imprinted pillars, and dewetted areas (low $h$ ). The interference colors in the
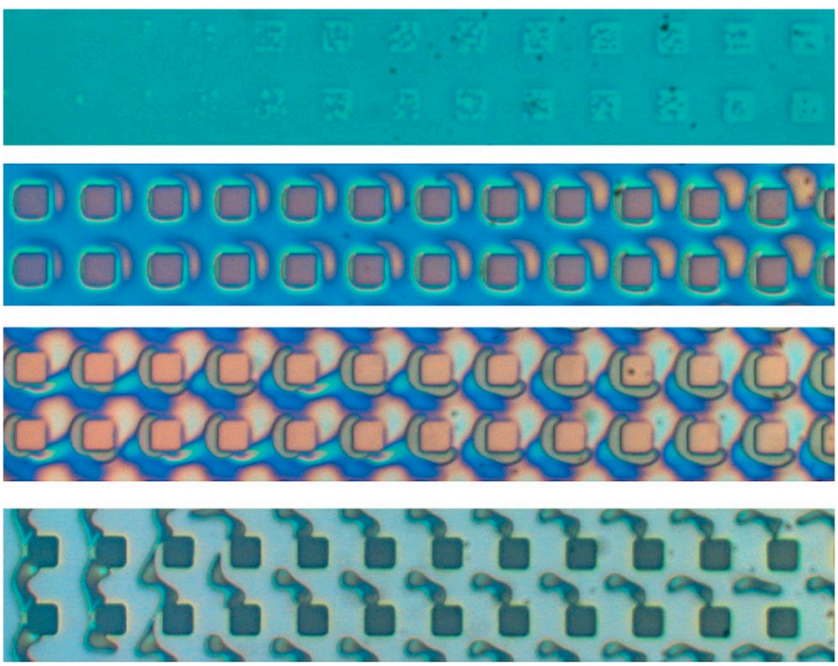

FIG. 3. Optical micrographs taken from a $300 \mathrm{~nm}$ thick mr-I $9030 \mathrm{E}$ thermocurable resist $\left(t_{\text {imprint }}=90 \mathrm{~s}\right)$. The fast evolution of the imprint can be seen in the four rows. (1) It starts with capillary bridges below the stamp pillars, until holes are formed. (2) Then capillary bridges start from the recessed stamp areas around the pillars. (3) These capillary bridges draw material from intermediate areas (dewetting). (4) The voids try to minimize their surface. original (in the online edition of this article) can be attributed to the total range of thickness present in one imprint and can be considered as between initial 300 and $75 \mathrm{~nm}$ below the pillars. As can be seen in Fig. 4, the partial dewetting can even lead to thickness much lower than the residual layer thickness.

Apart from this, viscous fingering phenomena can be observed, which have already been described in Ref. 1. This is the case if pressure is released while the resist is still soft and compressed air expands rapidly within the viscous resist, leading to a flower-like microstructure, e.g., Figs. 4(c) and 4(d) were taken in the vicinity of a dust particle near the center of a stamp, where a release of pressure causes an immediate lifting of the stamp. Interestingly, two different cases can be seen for imprint of a stamp with $400 \mathrm{~nm}$ period pillar array: a total vanishing of the hole-like structures and flowers with sharp borders to the already molded holes. The difference is probably only due to the fact that in the first case the expanding air squeezes between the polymer and the stamp and in the second case the air finds a channel within the polymer, leaving the already molded structures unobstructed. It is another sign of the dynamics behind the imprint process.

Figure 4(b) shows another interesting feature. Even in the areas where the stamp pillars touch the polymer surface first, flower-like microstructures can be seen. From the shape it cannot be explained whether it is the result of air inclusions leading to viscous fingering below the pillars or capillary bridges, which were created before the pillars were touching the polymer.

\section{DISCUSSION}

The main question is, how well the micrograph sequence is describing the true evolution of an imprint and whether our assumption of a pressure gradient as the main cause for this evolution is incomplete or even wrong. There are different sources of error.

(a) In an isothermal fast process with a process time of 


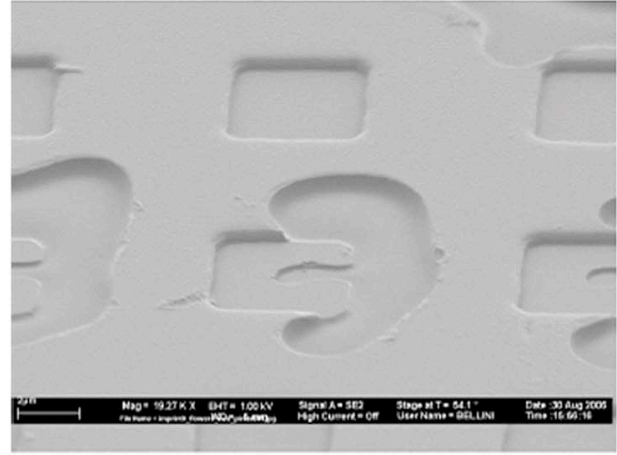

(a)

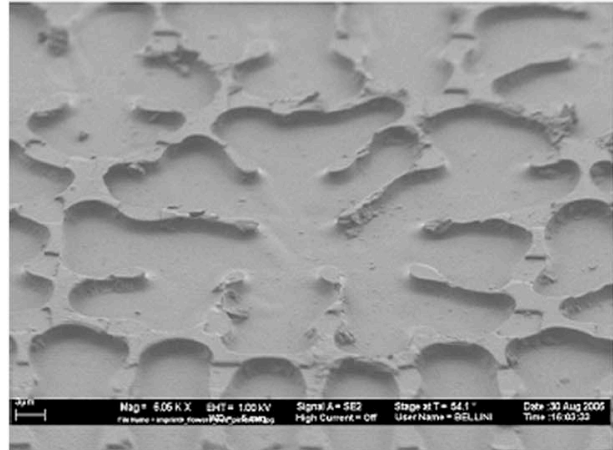

(c)

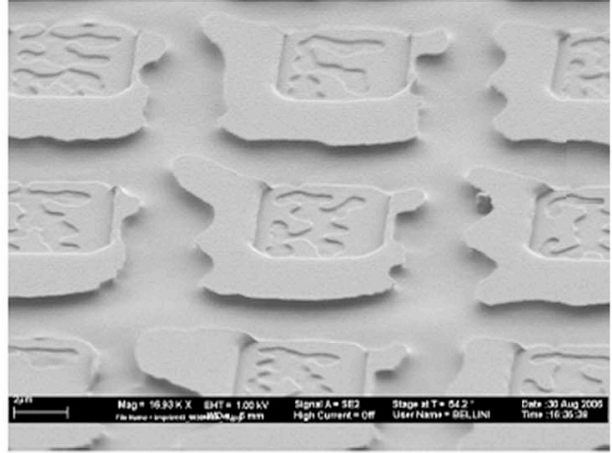

(b)

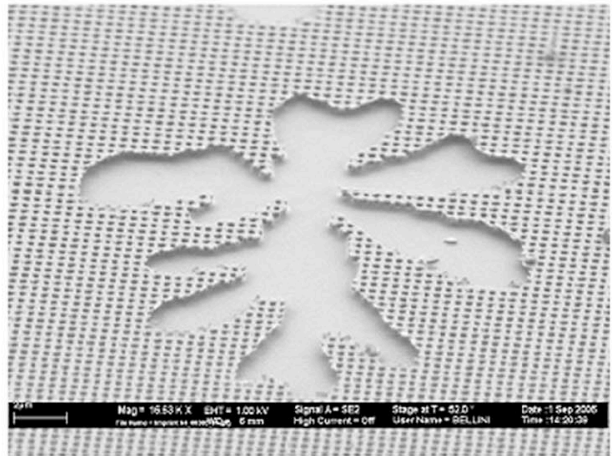

(d)
FIG. 4. Study of imprint artifacts. (a) Air bubble present after imprint. (b) Incomplete filling of the stamp cavities. (c) Flower-like artifacts due to expanding air. (d) A flower-like artifact in the middle of nanostructures. more than 1 min the freezing of imprint states must be almost instantaneous. Because in the micrographs sharp features can be seen, it is anticipated, that this is the case for the current setup. Otherwise the observed states of continuous imprint would be distorted or smeared out after pressure release. This can be the case by air expansion or continued flow during the time which is needed to release the pressure and separate the stack from the heated plates. The following rough estimations were done using textbook formulas and do not represent an exact modeling. They indicate that the time to heat a stack of two silicon wafers from 20 to $180{ }^{\circ} \mathrm{C}$ is small (less than $100 \mathrm{~ms}$ ) in comparison to the minimum mold filling time of about $1 \mathrm{~s}$ [taken as a rough estimate calculated from Eq. (1) for linear gratings in the area of highest pressure], but the time to cool it in air (from 180 to $80^{\circ} \mathrm{C}$ ) has to be considered as similar. We believe that the main reason for the instant freezing is that the molding in areas with reduced pressure is much slower than in the areas of high pressure. Therefore, not only the cooling, but also the instant pressure release causes the polymer to stop flowing.

(b) Furthermore, the influence of other factors such as a temperature gradient cannot be excluded totally. In the present setup, this is considered as negligible, simply because the heat transfer to the clamped areas is considered as fast in comparison to more than 1 min of intimate contact with the hot plates. However, as long as the time for cooling and pressure release cannot be determined more precisely, the current setup only gives a rough quantitative estimate of the local imprint pressure and temperature.

(c) In a movie, the viewer anticipates a linear time evolution, which would be the case in the actual setup if the pressure drop were linear in distance and radial in wafer symmetry. A movie is both very illustrative but could also be misleading, e.g., it is not evident whether the air inclusions are vanishing within a fraction of a second, as seen in the movie. At present we cannot prove that the gradients are linear. Therefore, we think that the main significance of the movie is not so much the quantitative than the qualitative evolution of the pattern formation during an imprint. The visualization of a seamless evolution shows that even the imprint of simple mold geometries can be complex and that dynamic effects such as mound formation and capillary action play a significant role during the filling of cavities. This is new and can be a valuable tool for process optimization with even incomplete knowledge of the current setup.

Because the micrographs are taken from demolded structures, which are easily accessible by optical microscopy, the interference color image can be used for a three-dimensional mapping of the topography of the polymer film. Equally, it is expected that the effect of dynamic mold bending can be elucidated. If these effects can be correlated with simulations, then structure filling can be tuned by clever design and choice of process parameters, particularly in view of a fast process for production. Then dynamic effects will play a 
prominent role and the clarification of their origin is crucial for the further process optimization. These dynamic effects can comprise the bending depending on the speed of polymer flow, the pressure buildup, dissolution of air, and noncomplete imprint in isothermal processes. In contrast to this, the method has some potential to investigate material parameters such as viscosity, if imprints of specific patterns are compared with simulations. The prerequisite for this is that nonlinear effects such as shear thinning are taken into account or excluded, and the effect of various gradients is quantified. Then the interchange of pressure and time, according to Stefan's equation, gives a sound representation of the real case. In the future the making of a movie from a single imprint could be done automatically by using sophisticated image processing. The evolution over a distance of $4 \mathrm{~mm}$, which is larger than typical bending lengths, ${ }^{7}$ shows that the method can probably also be used for periodic structures with sizes larger than $10 \mu \mathrm{m}$. These sizes apply for the current process parameters.

As stated before, with the current setup, the pressure release at high temperatures does not seem to lead to major defects or imperfections, as long as the imprint is complete and air is totally dissolved in the polymer. This has to be assured by a sufficient imprint time for all locations. The reason why viscous fingering did not occur as the normal case in the areas of large pressure inhomogeneity, e.g., created by the intentional gaps behind stamp and substrate, can be attributed to three reasons: (1) the partial evacuation of the chamber before the imprint, (2) the abrupt stopping of the sinking and the constant gap $(h)$ due to capillary forces and the sufficient load of the clamps to avoid premature demolding, and (3) and the fast freezing of the structures due to the lifting of the stack from the hot plate. However, the isothermal setup is more susceptible to these effects than in the case, where the polymer is allowed to harden before the stamp is demolded, particularly in areas where dust particles create large gaps between stamp and polymer. This can be reduced, when the imprint chamber is evacuated (to about 5 mbar) before the imprint.

\section{CONCLUSION}

The ideal way to elucidate the polymer behavior would be to observe the formation of patterns in real time through a transparent stamp, as was done for large areas in Refs. 8 and
9. For control of single dimensional parameters, such as in situ measurement of the residual layer thickness, capacitive and scatterometry methods have been developed, ${ }^{10,11}$ which both integrate over large areas. If process times are further reduced, then local measurements of pressure and temperature will be necessary. Then it is likely that also the dynamics of the imprint of nanostructures can be observed. In contrast to this a method, using standard opaque stamps made from silicon and analyzing a defined gradient of an imprint parameter after a single imprint, would be more appropriate for current presses. Furthermore, it can be scaled down to submicron dimensions, simply by taking high resolution scanning electron microscopy or atomic force microscopy images.

Although in the HEX03 adapter the pressure gradient was realized using a nonperfect heating plate below the substrate, the design of a plate with a defined pressure gradient is likely to look similar, simply by voids with different dimensions. An alternative would be to etch these voids into the backside of a substrate or stamp. Even if this method does not prove to be suitable for different structure sizes, periodicities, parameters for substrate, resist thickness, and pattern height, it can be a good starting point for a better evaluation of dynamic effects during the filling of cavities in nanoimprint.

${ }^{1}$ H. Schift, L. J. Heyderman, M. Auf der Maur, and J. Gobrecht, Nanotechnology 12, 173 (2001).

${ }^{2} \mathrm{H}$. Schift and L. J. Heyderman, in Alternative Lithography-Unleashing the Potential of Nanotechnology, edited by C. Sotomayor Torres and D. J. Lockwood (Kluwer Academic, Dordrecht, 2003), Chap. 4, pp. 46-76.

${ }^{3}$ H. Schift and A. Kristensen, in Handbook of Nanotechnology, 2nd ed., edited by B. Bhushan (Springer, Berlin, 2007), pp. 239-278.

${ }^{4}$ H. Schift, L. H. Schift, S. Bellini, J. Gobrecht, F. Reuther, M. Kubenz, M. B. Mikkelsen, and K. Vogelsang, Microelectron. Eng. 84, 932 (2007).

${ }^{5}$ H. Schift, S. Park, and J. Gobrecht, J. Photopolym. Sci. Technol. 16, 435 (2003).

${ }^{6}$ ImageJ, Free Java image process and analysis tool, http://rsb.info.nih.gov/ ij/.

${ }^{7}$ L. J. Heyderman, H. Schift, C. David, J. Gobrecht, and T. Schweizer, Microelectron. Eng. 54, 229 (2000).

${ }^{8}$ L. G. Baraldi, Ph.D. thesis, ETH, Zurich, 1994, in German.

${ }^{9}$ J. M. Stauffer, Y. Oppliger, P. Regnault, L. Baraldi, and M. T. Gale, J. Vac. Sci. Technol. B 10, 2526 (1992).

${ }^{10}$ H. Hocheng and C. C. Nien, Jpn. J. Appl. Phys., Part 1 45, 5590 (2006).

${ }^{11}$ Z. Yu, H. Gao, and S. Y. Chou, Appl. Phys. Lett. 85, 4166 (2004). 